\title{
Magnetic field in active regions of the sun at coronal heights
}

\author{
V. M. Bogod ${ }^{1}$ and L. V. Yasnov ${ }^{2}$ \\ ${ }^{1}$ Special Astrophysical Observatory, St. Petersburg, Russia \\ email: vbog_spb@mail.ru \\ ${ }^{2}$ Radiophysical Research Institute, St.-Petersburg State University, Russia \\ email: Yasnov@pobox.spbu.ru
}

\begin{abstract}
A method is developed for estimation of the vertical structure of the magnetic field in active regions using multi-wave spectral-polarization measurements of radio waves which gives not only the dependence of magnetic field strength on height but also determines twodimensional form of a magnetic flux tube, emitted in the microwave range of wavelengths.
\end{abstract}

Keywords. Sun: radio radiation Methods: data analysis

\section{Introduction}

In Akhmedov et al. (1982) it was shown that the magnetic field strength measured for polarized sources above sunspots go down in the transition region by only $20 \%$. Attempts to analyze the structure of magnetic fields using radio data were undertaken many times (Golubchina et al. (1981), Mursh \& Hurford (1982), Lang \& Wilson (1983), Shibasaki (1986), Aschwanden \& Bastian (1994), Brosius \& White 2006). But the measurements were made with a limited number of wavelengths, which restricted the capabilities of this method.

In this study we use the observational results made with the RATAN-600 radio telescope applying the broadband polarization spectrograph which has a large number of channels (39) from $1.7 \mathrm{~cm}$ to $15 \mathrm{~cm}$ (Bogod et al. 1999). Owing to the use of multi-wave data, it is possibile to study the detailed altitude structure of the magnetic field. On the other hand, the reliability of determination of such a structure increases.

\section{Technique of determination of the structure of magnetic field}

We use of polarization of radio sources (instead of their intensity). This gives the chance, to a certain degree, to eliminate the interfering influence on the result of adjacent structures of the magnetic field in the active region.

The aim of solar scans processing was to determine the time dependence of the position of a chosen feature in polarized emission of an active region $-x_{\text {rad }}$. Changes in the position of a chosen feature of a spot on the photosphere for a given time interval were corrected using the parallactic angle and the angle of inclination of the Sun's axis in the ecliptic coordinate system.

Using heliolatitude $\varphi$ of a measured feature of AR, we calculate its location $x_{\text {calc }}\left(h, \lambda, t_{i}\right)$ on the solar disk in the coordinate system of the radio scan. We have used the known time dependence of heliolongitude of the sources on the photosphere

$$
\lambda\left(t_{i}\right)=\left(14.35-2.77 \sin ^{2}(\varphi)-0.9856\right) t_{i}+\lambda,
$$

where $\lambda$ - the constant longitude characterizing the source position. The adequacy of this dependence to the real position of the active region features under study was verified, 
which showed a high accuracy of calculations limited by the errors of coordinate readout in observational data. Next, we minimized the expression

$$
\sum_{i=1}^{N}\left(x_{\text {calc }}\left(h, \lambda, t_{i}\right)-x_{\text {rad }}\left(t_{i}\right)\right)^{2},
$$

where $N$ is the number of data series used (from 2 to 5 ), and determined the height above photosphere $h$ and $\lambda$. Such calculation was made for every wavelength. We believed that the polarized emission of radio sources is determined by cyclotron radiation at the third harmonics of the gyrofrequency.

Because of some uncontrollable errors of the irradiator installation probably occurrence of a systematic error at height definition for the given method. For reduction of this error to the minimum value and for obtaining uniform data the height of the maximum magnetic field magnitude is set equal to the height using model calculations. As it is seen from the subsequent figures, the magnetic field is located low enough in the solar atmosphere. For this reason the usage of model extrapolations does not give essential errors for its definition.

We shall take advantage of one-dimensional dipole approximation of a magnetic field (Takakura 1972)

$$
B=\frac{B_{o}}{(1+h / d)^{3}},
$$

where $B_{o^{-}}$the magnetic field strength at the level of the photosphere, $d$ - the depth of a dipole under photosphere ( $d \approx r$ - the radius of a spot), $h$ - the height above photosphere. On SOHO MDI magnetograms we define $B_{o}$ and $r$. Knowledge of the magnetic field strength in the lowest point for a given structure (at the highest frequency) $B$, could help us define its height. The average height from the two adjacent day measurements was calculated. The dispersion in definition $h$ is specified in the figures in the form of vertical line in a point of the maximum magnetic field. Practically in all cases as it is visible from the subsequent figures, the dispersion was insignificant.

\section{Magnetic field in active regions at coronal heights}

The developed method was applied to observations of various active regions. Let us consider some results. Figure 1 presents the results of measurements of the magnetic field altitude structure for the AR NOAA 0933 observed on 7-8 January 2007. During the same period the AR NOAA 0935 was observed. Magnetic structure of this region for 3-4 January 2007 is represented in Figure 2.

It is shown in Figure 2, that the magnetic flux tube for AR NOAA 0935 is directed upwards with some bends. It can be a projection of the three-dimensional screw structure of the magnetic field to a plane. Such structure is not unique.

\section{Discussion}

The height of the corona base in an active region for various models is about 2-4 Mm. From the paper of Gary (2001) it follows (in the case of the potential extrapolation of a photospheric magnetic field), that at these heights $B=400-700 \mathrm{G}$, if at the photosphere $B_{o}=3000 \mathrm{G}$. In our measurements a magnetic field strength in the transition region and the bottom of corona falls no more than by $20 \%$, that is, up to $B \approx 2400 \mathrm{G}$, if a field at the photosphere is $B_{o}=3000 \mathrm{G}$. 

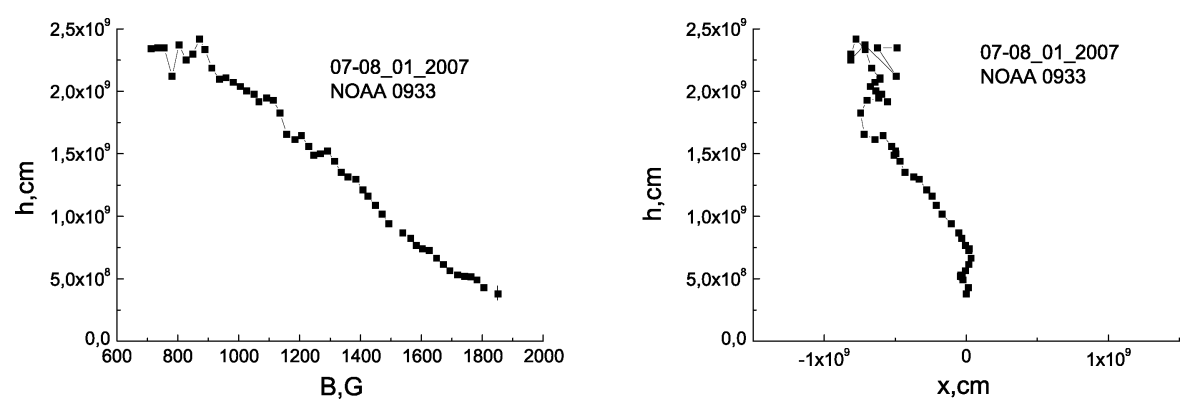

Figure 1. The results of calculations for AR NOAA 0933, observed on 7-8 January 2007. Dependence of the magnetic field with height - on the left and with the structure of the magnetic field - on the right. The value $x=\left(\lambda(f)-\lambda\left(f_{\max }\right)\right) \cos (\varphi) / 360 \times 43 \cdot 10^{10}$, where $f$-the frequency, $f_{\text {max }}$ - the frequency at which the magnetic field strength is maximum.
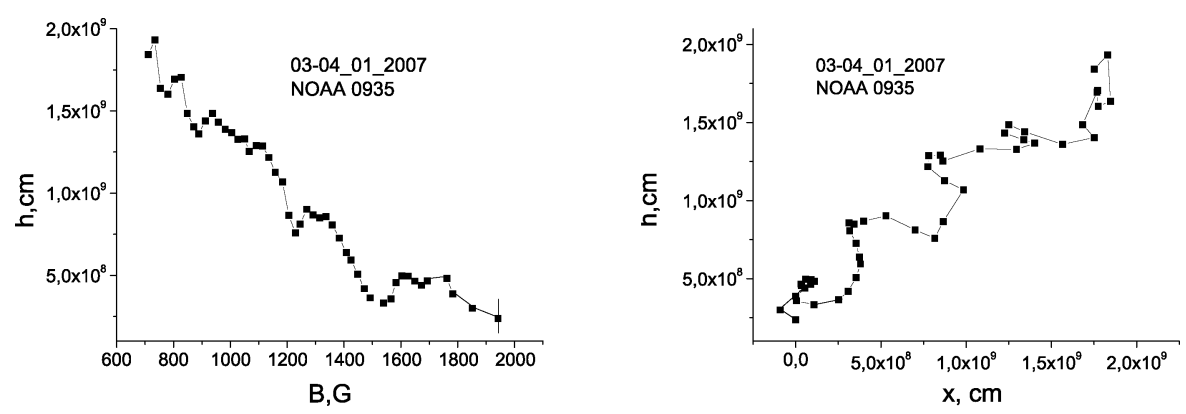

Figure 2. The structure of the magnetic field for AR NOAA 0935, observed on 3-4 January 2007.

Let us give additional arguments in favour of that magnetic fields extend highly in the corona. The loops radiated in soft X-ray, tend to be wider in their tops only by $30 \%$ than in their bases (Klimchuk 2000). The loops observed in lines 171 and $195 \dot{A}$ have still smaller expansion to tops: $0 \%$ for loops without flares and $13 \%$ for flare loops (Watko \& Klimchuk 2000). Expansion of loops unequivocally reflects a degree of decrease of the magnetic field strength along a loop. The specified expansion of ultraviolet loops should lead to decrease for the magnetic field in the loop top in comparison with its basis to $0 \%-27 \%$.

The presence of poorly diverging magnetic flux tubes can be justified theoretically by the presence in them of strong torsion of the magnetic field. In this case the field should be compressed (Zweibel \& Boozer (1985), Robertson et al. 1992) and tube expansion should decrease and magnetic field strength should increase.

The loop lengths investigated in the paper by Klimchuk (2000) are from $5 \cdot 10^{9} \mathrm{~cm}$ up to $33 \cdot 10^{9} \mathrm{~cm}$. That is, the magnetic field strength in the hottest parts of magnetic flux tubes (radiating in lines 171 and $195 \mathrm{~A}$ ) should not decrease in their tops, that is, at heights up to $1.5 \cdot 10^{10} \mathrm{~cm}$, more than for $30 \%$. Our measurements indicate that the magnetic field strength changes with height more considerably but it is essential more slowly than for theoretical computational models. Let us consider an elementary extrapolation of a magnetic field to the corona, using the established fact that the field in transition region should have the value not less than $80-85 \%$ of a photosphere field. We shall take advantage as above of one-dimensional dipole approximation of a magnetic field (Takakura (1972)). We shall estimate the value d, proceeding from a vertical gradient of a magnetic field on the photosphere. This gradient equals $0.1 \mathrm{G} / \mathrm{km}$ according to Severny 
(1965), 0.1-0.2 G/km (Rayrole \& Semel (1970)) and 0.3-0.5 G/km (Ioshpa \& Obridko (1965)). From $(2.3)$ we have $\dot{B}=3 B_{o} / d$. Then for $\dot{B}=0.1 \mathrm{G} / \mathrm{km}$ and $B_{o}=3000 \mathrm{G}$ we obtain $d=90000 \mathrm{~km}$, for $\dot{B}=0.4 \mathrm{G} / \mathrm{km}$ and $B_{o}=3000 \mathrm{G}$ we obtain $d=22500 \mathrm{~km}$. Thus the magnetic field strength of $1000 \mathrm{G}$ should be at heights from $10 \mathrm{Mm}$ up to 40 $\mathrm{Mm}$, that corresponds to our measurements.

\section{Conclusions}

1. A method is developed for the estimation of the vertical structure of the magnetic field in active regions using multi-wave spectral-polarization measurements of radio waves which gives not only the dependence of the magnetic field strength with height but also determines the two-dimentional form of a magnetic flux tube, emitted in a microwave range of wavelengths.

2. Magnetic fields with a strength of about $600 \mathrm{G}$ are located at sufficiently large altitudes in the solar atmosphere (up to $25000 \mathrm{~km}$ ), which well confirms the ultraviolet and X-ray observations, according to which the divergence of field tubes is small.

3. The topology of a magnetic flux tube emitted at microwaves in some investigated cases can have screw structure.

\section{References}

Akhmedov, S. B., Gelfreikh, G. B., Bogod, V. M., \& Korzhavin, A. N. 1982, Solar Phys., 79, 41 Golubchina, O. A., Ikhsanova, V. N., Bogod, V. M., \& Golubchin, G. S. 1981, Solnechnye Dannye, 4, 108 (in Russian)

Mursh, K. A. \& Hurford, G. J. 1982, ARAA, 20, 497

Lang, K. A. \& Wilson, R. F. 1983, Adv. Sp. Res., 11, 2

Shibasaki, K. 1986, ApESSS, 119, 21

Aschwanden, M. J. \& Bastian, T. S. 1994, ApJ, 426, 425

Brosius, J. W. \& White, S. M. 2006, ApJ, 641, L69

Bogod, V. M., Garaimov, V. I., Komar, N. P., \& Korzhavin, A. N. 1999, Proc. 9th European Meeting on Solar Physics, (ESA, SP-448), p. 1253

Takakura, T. 1972, Solar Phys., 26, 151

Gary, G. A. 2001, Solar Phys., 203, 71

Rayrole, J. \& Semel, M. 1970, A\&̈A, 6, 288

Robertson, J. A., Hood, A. W., \& Lothian, R. M. 1992, Solar Phys., 137, 273

Zweibel, E. G. \& Boozer, \& A. H. 1985, ApJ, 295, 642

Klimchuk, J. A. 2000, Solar Phys., 193, 53

Watko, J. A. \& Klimchuk, J. A. 2000, Solar Phys., 193, 77

Ioshpa, B. A. \& Obridko, V. N. 1965, Solnechnye dannye, 5, 62 (in Russian)

Severny, B. 1965, Izv. Crimea. astr. obs., 33, 34 (in Russian) 